\title{
The $\mathrm{Ca}^{2+}$-Regulated Photoprotein Obelin as a Tool for SELEX Monitoring and DNA Aptamer Affinity Evaluation
}

\author{
Vasilisa V. Krasitskaya ${ }^{1} *$ D, Natalia S. Goncharova ${ }^{2}$, Vladislav V. Biriukov ${ }^{2}$, Eugenia E. Bashmakova ${ }^{1}$, \\ Marsel R. Kabilov ${ }^{3}$, Ivan K. Baykov ${ }^{3}$, Aleksey E. Sokolov ${ }^{2,4}$ and Ludmila A. Frank ${ }^{1,2}$ \\ ${ }^{1}$ Institute of Biophysics SB RAS, Federal Research Center KSC SB RAS, Krasnoyarsk, Russia \\ ${ }^{2}$ Siberian Federal University, Krasnoyarsk, Russia \\ ${ }^{3}$ Institute of Chemical Biology and Fundamental Medicine SB RAS, Novosibirsk, Russia \\ ${ }^{4}$ Kirensky Institute of Physics, Federal Research Center KSC SB RAS, Krasnoyarsk, Russia
}

Received 11 December 2019, accepted 29 March 2020, DOI: 10.1111/php.13274

\begin{abstract}
Bioluminescent solid-phase analysis was proposed to monitor the selection process and to determine binding characteristics of the aptamer-target complexes during design and development of the specific aptamers. The assay involves $\mathrm{Ca}^{2+}$-regulated photoprotein obelin as a simple, sensitive and fast reporter. Applicability and the prospects of the approach were exemplified by identification of DNA aptamers to cardiac troponin I, a highly specific early biomarker for acute myocardial infarction. Two structurally different aptamers specific to various epitopes of troponin I were obtained and then tested in a model bioluminescent assay.
\end{abstract}

\section{INTRODUCTION}

Nucleic acid aptamers are a unique class of affinity ligands that offer significant advantages over antibodies, among which smaller size, greater stability and ease of production by chemical synthesis should be noted $(1,2)$. These single-stranded DNA or RNA molecules (typically 25-100 nt), with a particular three-dimensional shape, are capable of binding a wide variety of targets beginning from metal ions to whole cells with a high affinity (KD ranges from $10^{-9}$ to $10^{-12} \mathrm{M}$ ), specificity and selectivity $(3,4)$. Aptamers are mainly isolated from large random libraries through systematic evolution of ligands by exponential (SELEX) enrichment. Several techniques were developed to monitor SELEX. Among those are the ones based on separation techniques (dialysis, ultrafiltration, electrophoresis or HPLC) and mixture-based techniques (fluorescence intensity and anisotropy, UV-Vis absorption and circular dichroism, surface plasmon resonance, etc.) (see, e.g. 5). Besides, there are widely used electrophoretic mobility shift assay (EMSA) (6), Differential Radial Capillary Action of Ligand Assay (DRaCALA) (7) and ELISAlike assay based on aptamer (ELASA) with fluorescent (8) or colorimetric (9) detection. Some of them are time- or cost-consuming, the other ones require complicated equipment and highly qualified staff.

In current work, we propose the photoprotein obelin as a label in ELASA binding assay for fast and easy determination of

*Corresponding author email: vasilisa.krasitskaya@ibp.ru (Vasilisa V. Krasitskaya) (C) 2020 American Society for Photobiology aptamer-target binding characteristics throughout the whole aptamer discovery process. It includes monitoring of DNA library enrichment in the course of selection, identification of the individual aptamers with high affinity and specificity to the target, optimization of the aptamers size and identification of the matched aptamer pairs.

The $\mathrm{Ca}^{2+}$-regulated photoprotein obelin is a stable noncovalent complex of an apoprotein and a pre-oxidized substrate, 2-hydroperoxycoelenterazine (10). Light is emitted as a fast blue flash $\left(\lambda_{\max }=482 \mathrm{~nm}\right)$ when calcium ions become bound to the protein. At saturating concentration of $\mathrm{Ca}^{2+}$, a linear relationship between protein amount and the emitted light integral intensity overlaps several logs of concentration. As a reporter, obelin has a number of advantages such as bioluminescence high quantum yield and virtual absence of the background that provide high sensitivity of the assay, simple reaction (no dependence on a substrate, oxygen or any cofactor), stability under storage and modifications, and no toxicity. All this makes obelin-based labels convenient for research and biomedical applications $(11,12)$.

Here, we describe the use of obelin as a highly sensitive reporter for monitoring the course of selection and evaluation of the affinity of DNA aptamers to cardiac troponin I (cTnI), a highly specific and early biomarker for acute myocardial infarction (13). Low diagnostic level of this cardiac marker $\left(0.01 \mu \mathrm{g} \mathrm{L}^{-1}\right)$ causes high demands on the detection techniques in terms of sensitivity. So, the development of novel supersensitive biosensors including aptasensors for cTnI detection is a challenge to modern biotechnology. Recently, several DNA aptamers were obtained to recombinant variant of cTnI (14-16). As opposed to the works mentioned, in our study a native human cardiac troponin I was used as a target for development of aptamers.

\section{MATERIALS AND METHODS}

The highly purified $\mathrm{Ca}^{2+}$-regulated photoprotein obelin with a unique cysteine residue (Obe D12C) was obtained as previously described (17). Coelenterazine was from NanoLight Technologies. Conjugate Obe D12C with monoclonal anti-cardiac troponin I antibody (Obe-anti cTnIAb) was synthesized as described in Ref. (18). Obe D12C conjugate with oligothymidine carrying a 5'-hexamethylenamino group 5'- $\mathrm{NH}_{2}-\left(\mathrm{CH}_{2}\right)_{6}$ $\mathrm{p}-\mathrm{T}_{30} \quad\left(\right.$ Obe- $\left.\mathrm{dT}_{30}\right)$ was synthesized as described in Ref. (19). Hybrid protein streptavidin-obelin (Stavi-Obe) was obtained as described in Ref. (20) 
Native human cardiac troponin I, recombinant cardiac troponin I, troponin $\mathrm{T}(\mathrm{cTnT})$ and troponin $\mathrm{C}(\mathrm{cTnC})$ were from HyTest (Finland). Streptavidin, human immunoglobulin G (hIgG) and bovine serum albumin (BSA) were from Sigma-Aldrich. Human myoglobin (Myo), creatine kinase MB (CKMB) and monoclonal anti-cardiac troponin I antibody (anti-cTnIAb) were from Abcam. Human serum albumin (HSA) was obtained from Reanal (Hungary), Hot Start Taq DNA polymerase-from Evrogen (Russia) and dNTPs - from SibEnzyme (Russia).

All oligonucleotides as well as their biotinylated derivatives were synthesized by Biosan (Russia). The ssDNA library was purchased from Sangon Biotech (China). The enriched library was sequenced on a MiSeq platform (Illumina) in SB RAS Genomics Core Facility (ICBFM SB RAS, Russia) as described in the Supporting Information. A solid-phase bioluminescent assay was carried out in white Low Volume Stripwell ${ }^{\mathrm{TM}}$ Microplate (Corning).

Selection of the aptamers specific to cTnI. Single-stranded DNA library consisting of a randomized region of 40 nucleotides $\left(\mathrm{N}_{40}\right)$ flanked by two constant primer hybridization regions for PCR (5'GGCAGCAGGAAGACAAGACA-N 40 -TGGTTCTGTGGTTGCTCTGT3') was used as the initial pool. Before each round of selection and binding experiment, the DNA library or individual aptamer was denatured by heating for $5 \mathrm{~min}$ at $90^{\circ} \mathrm{C}$ in binding buffer $(0.15 \mathrm{M} \mathrm{NaCl}$, $50 \mathrm{~mm}$ K-Na phosphate buffer $\mathrm{pH} 7.0$ (PBS), $1 \mathrm{~mm} \mathrm{MgCl}_{2}$ ) and then renatured at room temperature (RT) for $15 \mathrm{~min}$.

The cTnI or proteins used for negative selection-cTnT, cTnC, Myo, CKMB, HSA or BSA - were immobilized on the well surface by incubations $\left(50 \mu \mathrm{L}\right.$ per well, $10 \mu \mathrm{g} \mathrm{mL}^{-1}$ in PBS) overnight at $4^{\circ} \mathrm{C}$. After washing, $1 \mathrm{nmol}$ (rounds $1-3$ ) or $0.2-0.48 \mathrm{nmol}$ (rounds $4-12$ ) of ssDNA was added into the cTnI-coated well and incubated at the RT for $1 \mathrm{~h}$ (rounds $1-$ 3), $45 \mathrm{~min}$ (rounds 4-7) or $30 \mathrm{~min}$ (rounds 8-12) with shaking. The wells were washed from two to five times ( $0.1 \%$ Tween 20 in binding buffer), and the bound ssDNA was eluted three times with $50 \mu \mathrm{L}$ of TE buffer (10 mm Tris- $\mathrm{HCl} \mathrm{pH} \mathrm{8.0,1} \mathrm{mm}$ EDTA) at $95^{\circ} \mathrm{C}$ for $10 \mathrm{~min}$.

Negative selection was performed with HSA (rounds 3 and 9), cTnT (rounds 4 and 11), BSA (round 5), Myo (rounds 6 and 10), CKMB (round 7) and cTnC (rounds 8 and 12). The selection was strengthened by decreasing incubation time and increasing the number of washing steps.

The cTnI-binding aptamers were amplified using symmetric (a) and then asymmetric (b) PCR:

a. $5 \mu \mathrm{L}$ of the aptamer pool (5-15 ng) in TE was mixed with $45 \mu \mathrm{L}$ of symmetric PCR mixture containing PCR buffer, $0.2 \mathrm{~mm}$ dNTPs, forward (5'-GGCAGCAGGAAGACAAGACA-3') and reverse (5'-ACAGAGCAACCACAGAACCA-3') primers, $0.3 \mu \mathrm{M}$ each and $0.05 \mathrm{U} \mu \mathrm{L}^{-1}$ Hot Start DNA polymerase. Amplification was performed as follows: $2 \mathrm{~min}$ at $95^{\circ} \mathrm{C}, 15$ cycles of $30 \mathrm{~s}$ at $95^{\circ} \mathrm{C}, 15 \mathrm{~s}$ at $56^{\circ} \mathrm{C}$ and $15 \mathrm{~s}$ at $72^{\circ} \mathrm{C}$.

b. $5 \mu \mathrm{L}$ of the symmetric PCR product in TE buffer was mixed with $45 \mu \mathrm{L}$ of the asymmetric PCR mixture containing PCR buffer, $0.2 \mathrm{~mm}$ dNTPs, $1 \mu \mathrm{M}$ forward primer, $50 \mathrm{~nm}$ reverse primer and 0.05 $\mathrm{U} \mu \mathrm{L}^{-1}$ Hot Start DNA polymerase. Amplification was performed as described in (a).

For every next round of selection, ssDNA was cleaned with Amicon Ultra-0.5 10K Centrifugal Filter Devices (Merck Millipore, Germany). The concentration of ssDNA was quantified by NanoDrop Lite Spectrophotometer (Thermo Scientific). In total, 12 rounds of selection were carried out.

Biotinylated enriched DNA library was synthesized after 8, 10 and 12 selection rounds by amplification as described above using biotinylated forward primer (Bio-5'- GGCAGCAGGAAGACAAGACA-3').

Binding affinity studies of individual DNA aptamers by bioluminescent solid-phase assay. The $50 \mathrm{~nm}$ solution of biotinylated aptamer or biotinylated enriched DNA library $(50 \mu \mathrm{L})$ in binding buffer was added into the streptavidin-activated well, incubated with shaking for $30 \mathrm{~min}$ at RT and washed (three times, PBS, $1 \mathrm{~mm} \mathrm{MgCl}_{2}, 0.1 \%$ Tween 20). Then, $50 \mu \mathrm{L}$ aliquots of cTnI $(1.25 \mu \mathrm{M}-0.08 \mathrm{nM}$ in binding buffer $)$ or $50 \mu \mathrm{L}$ binding buffer (control wells) was added and incubated for $1 \mathrm{~h}$ at RT. After washing, $50 \mu \mathrm{L}$ aliquots of Obe-anti cTnIAb $\left(0.1 \mu \mathrm{g} \mathrm{mL}{ }^{-1}\right.$ in binding buffer containing $0.1 \%$ BSA) were added, incubated for $40 \mathrm{~min}$ at RT and washed thereafter. Obelin bioluminescence was measured with LB 940 Multimode Reader Mithras (Berthold, Germany) by rapid injection of $0.1 \mathrm{M} \mathrm{CaCl}_{2}$ in $0.1 \mathrm{M}$ Tris- $\mathrm{HCl}, \mathrm{pH} 8.8$. The signal was integrated for $5 \mathrm{~s}$.
Signals from the corresponding control wells were taken as the background and subtracted during dose-response curve plotting. All measurements were performed in duplicate. The dissociation constants (KD) were calculated by fitting the relative bioluminescence emission to Eq. (1), a slightly modified equation used to calculate immunoglobulins' affinity constant described elsewhere (21):

$$
\frac{L}{L_{\max }}=\frac{x+R+\mathrm{KD}-\sqrt{(x+R+\mathrm{KD})^{2}-4 x R}}{2},
$$

where $L$ is the integral light intensity from a sample; $L_{\max }$ is the integral light intensity from samples with saturating concentrations of cTnI; $R$ is the surface concentration of biotinylated aptamer; $x$ is concentration of cTnI in solutions placed into the wells; and KD is the dissociation constant. Parameters were calculated by nonlinear regression using SigmaPlot 2000 software.

DNA aptamers' specificity study. cTnI, cTnT, cTnC, HSA or hIgG $\left(50 \mu \mathrm{L}, 10 \mu \mathrm{g} \mathrm{mL}^{-1}\right.$ in PBS) was adsorbed on the surface of microtiter plates, overnight at $4{ }^{\circ} \mathrm{C}$. After washing, the solution of biotinylated aptamer (TnAp1, TnAp2 or TnAp12) in binding buffer was placed into the wells, incubated for $1 \mathrm{~h}$ at RT and washed (PBS, $1 \mathrm{~mm} \mathrm{MgCl}_{2}, 0.1 \%$ Tween 20). Then, $50 \mu \mathrm{L}$ hybrid protein Stavi-Obe solutions (50 ng mL ${ }^{-1}$ in binding buffer) were incubated for $40 \mathrm{~min}$ at RT. After washing, obelin bioluminescence was measured as described above.

Search of noncompetitive pairing aptamer for TnAp2t1 or TnAp2t3. The $50 \mathrm{~nm}$ solution of biotinylated aptamer (TnAp1, 2, 2t1, 2t2, 2t3, 4, $5,10,12,13,14)$ in binding buffer was placed into streptavidin-activated wells, incubated with shaking for $30 \mathrm{~min}$ at RT and washed (three times, PBS, $1 \mathrm{~mm} \mathrm{MgCl}_{2}, 0.1 \%$ Tween 20). The $50 \mathrm{~nm}$ solution of cTnI in binding buffer was placed into the wells and incubated for $1 \mathrm{~h}$ at RT. After washing, the $50 \mathrm{~nm}$ solution of TnAp2t1-(dA) 20 or TnAp2t3-(dA) 20 was added into the wells, incubated for $1 \mathrm{~h}$ at $\mathrm{RT}$ and washed. Then, $50 \mu \mathrm{L}$ aliquots of Obe- $\mathrm{dT}_{30}\left(0.5 \mu \mathrm{g} \mathrm{mL}^{-1}\right.$ in binding buffer, containing $0.1 \% \mathrm{BSA}$ ) were added, incubated for $40 \mathrm{~min}$ at $\mathrm{RT}$ and washed thereafter. Obelin bioluminescence was measured as described above.

Model aptamer-based solid-phase bioluminescent assay of cTnI. The $25 \mathrm{~nm}$ solution of biotinylated aptamer (TnAp1, 2, 4, 5, 10, 12) in binding buffer was placed into streptavidin-activated wells, incubated with shaking for $30 \mathrm{~min}$ at RT and washed (three times, PBS, $1 \mathrm{~mm}$ $\mathrm{MgCl}_{2}, 0.1 \%$ Tween 20). Then, the $50 \mu \mathrm{L}$ aliquots of cTnI (9-0.01 nм in binding buffer) were added into the wells and incubated for $1 \mathrm{~h}$ at RT. After washing the wells, the $50 \mathrm{~nm}$ solution of TnAp2t3-(dA) 20 was added into the wells, incubated for $1 \mathrm{~h}$ at RT and washed. Then, $50 \mu \mathrm{L}$ aliquots of Obe- $\mathrm{dT}_{30}\left(0.5 \mu \mathrm{g} \mathrm{mL}^{-1}\right.$ in binding buffer, containing $0.1 \%$ BSA) were added, incubated for $40 \mathrm{~min}$ at RT and washed thereafter. Obelin bioluminescence was measured as described above.

Model aptamer-based solid-phase bioluminescent assay of cTnI in human serum. Performed as described above, Bio-TnAp10 was immobilized on streptavidin-activated surface of a well, and then, aliquots of human serum of healthy patient containing 9-0.01 nM of cTnI were added into the wells with further addition of aliquots of TnAp2t3$(\mathrm{dA})_{20}$. The surface-formed complex was revealed by Obe- $\mathrm{dT}_{30}$ bioluminescence.

\section{RESULTS AND DISCUSSION}

In order to obtain high-affinity DNA aptamers to cTnI, the conventional SELEX technology was applied. Randomized oligonucleotide pool $\left(10^{14}-10^{15}\right.$ different sequences) was incubated with cTnI adsorbed on the Stripwell surface, unbound DNAs were washed out, and bound aptamers were eluted by denaturation. Every round of selection yielded the DNA mixture enriched with the affinity sequences binding to the target. To enhance the specificity of aptamer, we used human serum protein and other cardiac markers such as troponins $\mathrm{T}$ and $\mathrm{C}$, myoglobin and creatine kinase $\mathrm{MB}$ for negative selection steps (see Material and Methods section).

To monitor the selection course and to study the binding affinity of individual aptamer, we developed the bioluminescent 
solid-phase assay using a stable, "self-contained", triggerable and fast reporter- $\mathrm{Ca}^{2+}$-regulated photoprotein obelin.

With the earlier developed methods applied (17), we chemically conjugated obelin with monoclonal anti-cardiac troponin I antibody or with oligothymidine giving Obe-anti cTnIAb and Obe- $\mathrm{dT}_{30}$, correspondingly. Fig. 1 gives the relationship between signal intensity and concentration of these conjugates as compared to the one of the recombinant obelin. It is linear over 5-6 $\log$ units with the detection limit being about 1 attomol (calculated as a protein amount in a sample with a signal-to-background ratio of 2). To trigger obelin bioluminescence, nothing but $\mathrm{Ca}^{2+}$ ions are needed, and the stop-reaction stage is missing. Bioluminescent response is integrated within a few seconds after the reaction is triggered. This significantly distinguishes obelinbased labels from those based on peroxidase or fluorescent reporters (see, e.g. 7-9, 15) and makes them more convenient for applications.

Bioluminescent solid-phase assay to monitor DNA library enrichment and evaluate individual aptamers' affinity

The scheme for the proposed bioluminescent solid-phase assay is presented in Fig. 2a. Biotinylated derivatives of the enriched DNA library or individual aptamers were immobilized on the surface of microtiter plate activated with streptavidin. After incubation with cTnI, the complex formed on the surface was detected by the conjugate Obe-anti cTnIAb. Obelin bioluminescence was triggered by $\mathrm{Ca}^{2+}$ and measured by plate uminometer.

To evaluate DNA library enrichment with anti-troponin I aptamers, the libraries enriched after the 8th, 10th and 12th SELEX rounds were re-amplified with biotinylated forward primer and

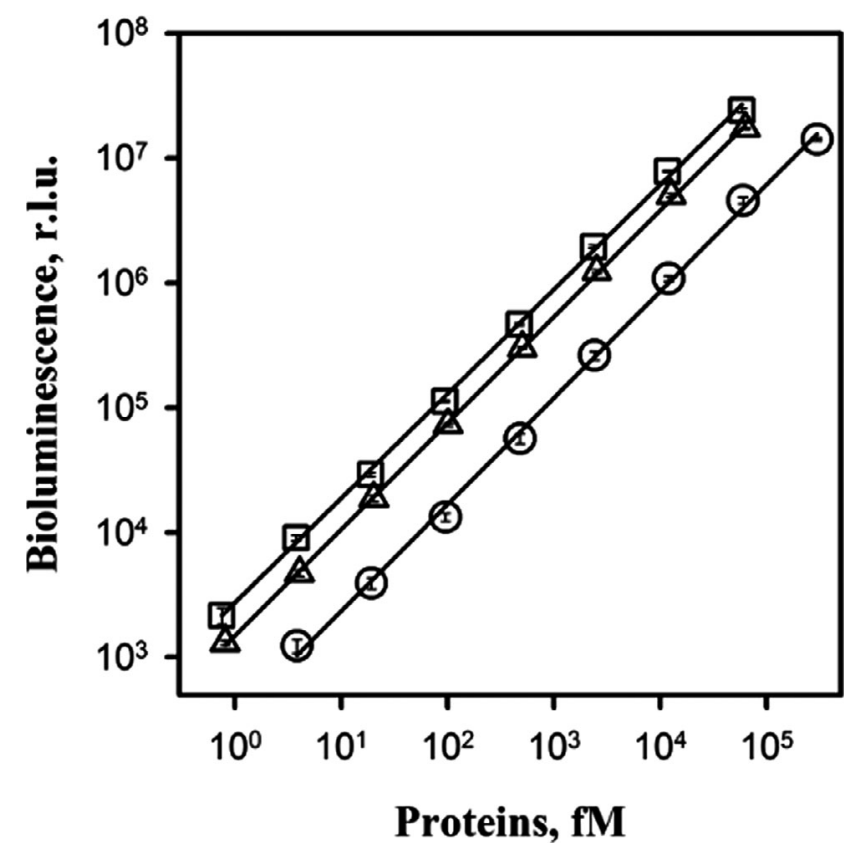

Figure 1. Bioluminescence as a function on the amount of obelin (-O-), Obe-anti cTnIAb $(-\Delta-)$ and Obe- $\mathrm{dT}_{30}(-\square-)$ conjugates. Each point is an average \pm 1 standard deviation $(\mathrm{n}=3)$.
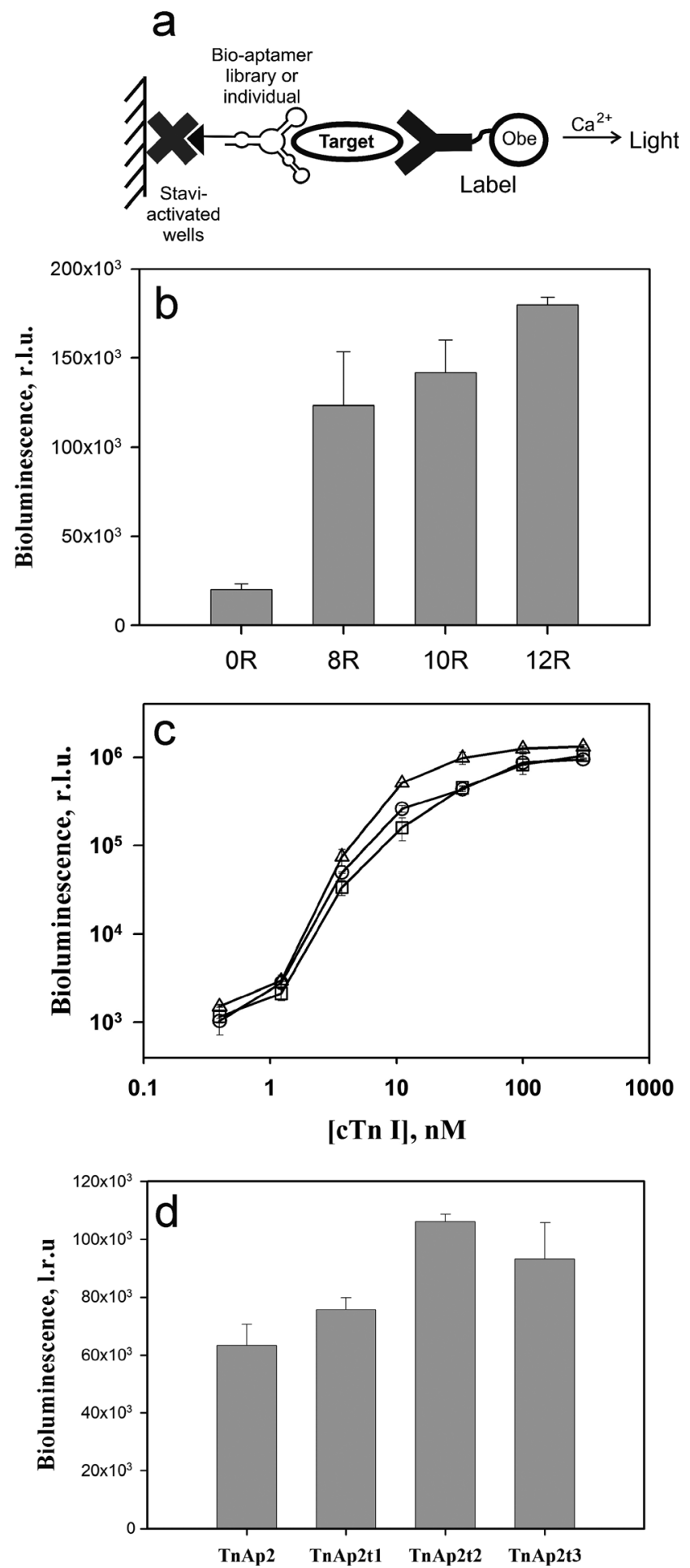

Figure 2. (a) Bioluminescent solid-phase assay to monitor the library enrichment and to evaluate the individual aptamers' affinity. Label - conjugate Obe-anti cTnIAb; Stavi-streptavidin. (b) Bioluminescent assay of initial DNA library (0R) and after SELEX rounds $8(8 \mathrm{R}), 10(10 \mathrm{R})$ and 12 (12R). (c) Saturation curve for aptamers TnAp1 (- $\square-$ ), TnAp2 (-O-) and TnAp12 (- $\Delta-)$ obtained by solid-phase bioluminescent assay. (d) Full-length aptamer TnAp2 and its truncated variants' binding ability to cTnI ( $33 \mathrm{~nm})$. All the points are the medium value from 3 independent measurements \pm standard deviation. 
Table 1. Nucleotide sequences of candidate DNA aptamers and some of their properties

\begin{tabular}{|c|c|c|c|c|}
\hline Aptamer & Nucleotide sequence 5 '-Bio $\rightarrow 3$ ' & Abundance, $\%$ & G \%; GQ motif* (+ or - ) & $\mathrm{KD}, \mathrm{nm}$ \\
\hline TnAp1 & $\begin{array}{l}\text { GGCAGCAGGAAGACAAGACATGGGTGGCGGGGACGGGGC } \\
\text { GATGGGAACTTAGATTGCTAGTGGTTCTGTGGTTGCTCTGT }\end{array}$ & 17.64 & $40(+)$ & 61.51 \\
\hline TnAp2 & $\begin{array}{l}\text { GGCAGCAGGAAGACAAGACAGGCAGTGTCACGCGCTCAAG } \\
\text { GGTGGAGGGGTCGGGGAGGTTGGTTCTGTGGTTGCTCTGT }\end{array}$ & 11.17 & $42.5(+)$ & 42.01 \\
\hline TnAp4 & $\begin{array}{l}\text { GGCAGCAGGAAGACAAGACACAACGCATGGGTGGGACGA } \\
\text { CGGGTGGGCAAGAGACACGCCTGGTTCTGTGGTTGCTCTGT }\end{array}$ & 6.97 & $38.8(+)$ & 167.1 \\
\hline TnAp5 & $\begin{array}{l}\text { GGCAGCAGGAAGACAAGACACACGGGAGGGAGGGTAGGG } \\
\text { TGTGTGTCGAATCACTGCGCATGGTTCTGTGGTTGCTCTGT }\end{array}$ & 5.23 & $41.3(+)$ & 255.7 \\
\hline TnAp10 & $\begin{array}{l}\text { GGCAGCAGGAAGACAAGACACCACATCTATGGGTGGGACG } \\
\text { ATGGGTGGGCCGAAACGACCTGGTTCTGTGGTTGCTCTGT }\end{array}$ & 1.98 & $36.3(+)$ & 121.4 \\
\hline TnAp12 & $\begin{array}{l}\text { GGCAGCAGGAAGACAAGACATCGGGAGGGAGGGAGGGC } \\
\text { AGTCTAGTCTCATGTGTTTCCATGGTTCTGTGGTTGCTCTGT }\end{array}$ & 1.2 & $37.5(+)$ & 24.16 \\
\hline TnAp13 & $\begin{array}{l}\text { GGCAGCAGGAAGACAAGACACCACGACCACCAACAGATCC } \\
\text { ACTAGCTATTCGACTGCACTTGGTTCTGTGGTTGCTCTGT }\end{array}$ & 1.1 & $23.8(-)$ & $-\dagger$ \\
\hline TnAp14 & $\begin{array}{l}\text { GGCAGCAGGAAGACAAGACACTACCCATACACTTAGGGA } \\
\text { CGGGTGGCCGGGGAGGGAGGTTGGTTCTGTGGTTGCTCTGT }\end{array}$ & 1 & $38.8(+)$ & 79.04 \\
\hline TnAp2t1 & GGCAGTGTCACGCGCTCAAGGGTGGAGGGGTCGGGGAGGT & & $52.5(+)$ & $39.06(20 \$)$ \\
\hline $\operatorname{TnAp} 2 \mathrm{t} 2$ & $\begin{array}{l}\text { AGACAAGACAGGCAGTGTCACGCGCTCAAGGGTGGAGGG } \\
\text { GTCGGGGAGGTTGGT }\end{array}$ & & $46.3(+)$ & 24.93 \\
\hline TnAp2t3 & GCTCAAGGGTGGAGGGGTCGGGGAGGT & & $59.3(+)$ & 30.6 \\
\hline
\end{tabular}

*The secondary structures of aptamers predicted by VIENNA RNA fold. ${ }^{\dagger}$ We failed in calculating the constant, probably due to the common epitope for binding of the labeled antibody and this aptamer. ${ }^{*} \mathrm{KD}$ was calculated by SPR, see Fig. S2.

the obtained biotinylated derivatives were analyzed by the proposed assay. The results of the bioluminescent assay are shown in Fig. 2b. The progressive increase in obelin bioluminescence with the number of rounds indicates the enrichment of the library with oligonucleotide sequences binding to cTnI.

After the 12th round of SELEX, the library was sequenced with the Illumina NGS. To choose potentially high-affinity aptamers, raw NGS data were analyzed as described in the Supporting Information. All sequences were ranked according to their abundance in the enriched library. The sequences of candidate aptamers and some of their properties are represented in Table 1.

To evaluate binding affinities of individual aptamers (TnAp1, $2,4,5,10,12,13$ and 14) applying the developed bioluminescent solid-phase assay, candidate aptamers bearing biotin at 5, terminus were chemically synthesized. Using the bioluminescent assay data, the saturation curves were plotted (Fig. 2c) and dissociation constants of aptamer-cTnI complexes (KD) were calculated. The highest binding affinity to cTnI was found for the most abundant aptamers TnAp1 and TnAp2, and for nonabundant aptamer TnAp12 (Table 1).

Specificity of aptamers TnAp1, TnAp2 and TnAp12 was evaluated for cTnI, cTnT, cTnC, HSA and hIgG by bioluminescent solid-phase assay according to the scheme in Fig. 3. Proteins under study were immobilized on the plate and incubated with biotinylated aptamer. The surface-formed complex was detected by using streptavidin-obelin hybrid protein. As is displayed in Fig. 3, the bioluminescent signals from nontarget proteins are lower than that from cTnI by more than an order of magnitude.

\section{Optimization of aptamer size}

Aptamer sequence is usually optimized by truncation so to narrow down the aptamer sequence region responsible for target binding. On the one hand, it reduces the cost of aptamer production, on the other-may increase the binding affinity. The truncations of aptamer TnAp2 were performed based on its 2D structure (see Supporting Information). All the truncated aptamer variants were biotin-labeled at the $5^{\prime}$-end and tested for their functionality using the developed bioluminescent binding assay. Deletion of primer sequences at $3^{\prime}$ and $5^{\prime}$ termini (TnAp2t1) was found to slightly enhance the affinity of the aptamers to cTnI (Fig. 2d). The binding ability of truncated aptamers TnAp2t2 and TnAp2t3 became essentially improved (Table 1). So, the 80nt long aptamer TnAp2 can be shortened down to $27 \mathrm{nt}$ with binding ability to cTnI improved at that.

It was interesting to evaluate aptamer binding affinity using another analytical approach. To make this experiment, we use recombinant variant of cTnI as a target. Aptamer's TnAp2t1 affinity was determined in parallel with the bioluminescent and surface plasmon resonance (SPR) techniques. Detailed

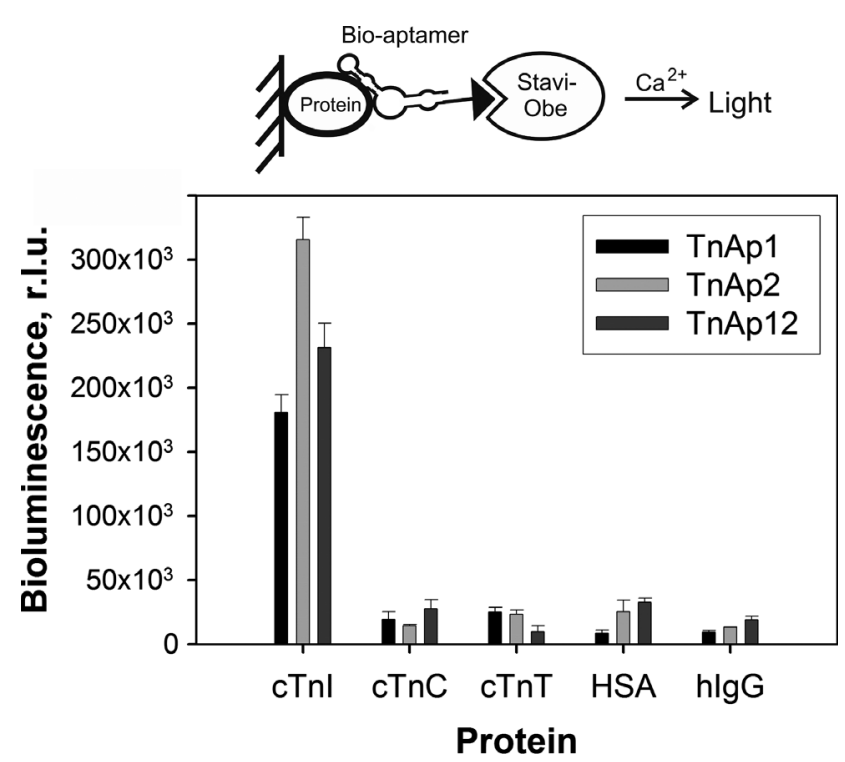

Figure 3. Scheme (upper panel) and results (lower panel) of the bioluminescent solid-phase assay of specificity of aptamers TnAp1, TnAp2 and TnAp12 toward troponin I and other proteins. 
description of the SPR experiment is given in the Supporting Information. The dissociation constant values for interaction of TnAp2t1 with the recombinant cTnI obtained using bioluminescent and SPR techniques were 96.5 and $20 \mathrm{~nm}$, correspondingly (Fig. S2). Thus, the assessment of the aptamer affinity by the proposed solid-phase bioluminescent method gives adequate results. The discrepancy in absolute values of the constant can be explained by differences in analysis conditions.

The screening of the aptamers bound to the distinct epitopes on cTnI

The pairs of aptamers that recognize distinct epitopes on the target protein can greatly improve the sensitivity and specificity of
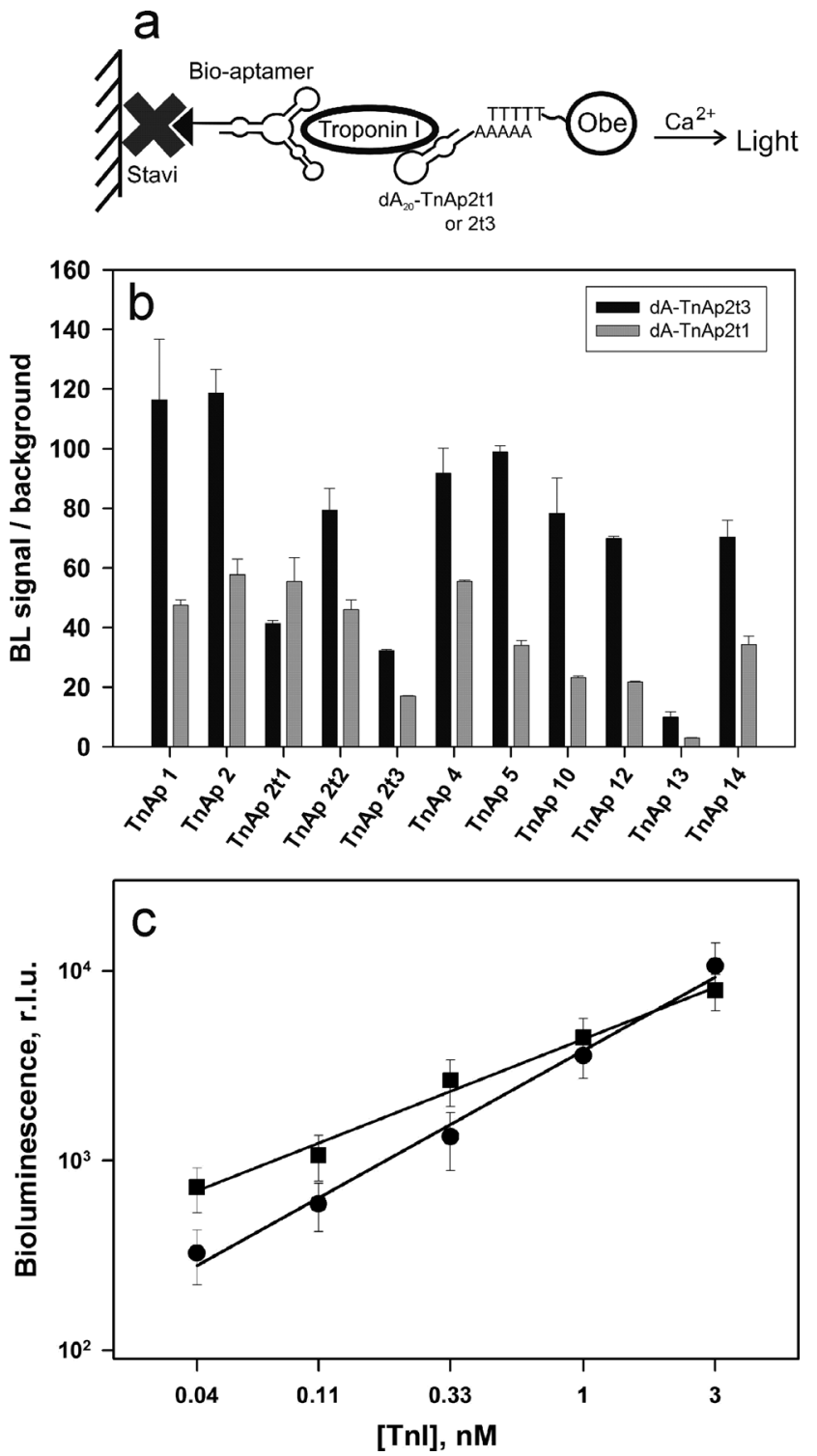

Figure 4. Scheme (a) and results (b) of the bioluminescent assay for identification of aptamers that form sandwich complexes with cTnI and TnAp2t1 or with cTnI and TnAp2t3. (c) Results of the model sandwichtype aptamer-based solid-phase bioluminescent assay of cTnI in buffer (-) or in human serum (-ם-) using aptamers Bio-TnAp10 and TnAp2t3 $(n=3)$. molecular detection. A simple experiment on competitive binding of cTnI by truncated variants of aptamer (TnAp2t1 or TnAp2t3) and other anti-cTnI aptamers allowed estimating whether all of them bind the same or different epitopes on a target. This analysis was carried out according to the scheme presented in Fig. 4 a. Biotinylated aptamer under study was immobilized on streptavidin-activated surface and incubated with cTnI. The truncated TnAp2t1 or TnAp2t3 carrying oligoadenylate at 3' terminus (TnAp2t1-dA 20 or TnAp2t3-dA 20 ) forms a sandwich-type complex only if aptamers are bound to different cTnI epitopes. This complex is detected by bioluminescent signal of the conjugate Obe- $\mathrm{dT}_{30}$. Thus, the increase in bioluminescence signal points to the formation of independent sandwich-type complex.

It is quite reasonable that the shortest aptamer TnAp2t3 (27 nt) forms a more stable triple complex as compared to TnAp2t1 (40 nt) due to the smaller space it occupies (Fig. 4b). Aptamers TnAp1, TnAp2, TnAp4, TnAp5, TnAp10 and TnAp12 yielding the highest bioluminescent response when being paired with aptamer TnAp2t3 were then tested in model bioluminescent assay of cTnI. The best result was obtained for the TnAp10 case: the linear dependence cTnI concentration-bioluminescent signal value was observed in the range of 3-0.04 nM. Close results were obtained when this pair of aptamers was applied to analyze cTnI in artificial human serum (Fig. 3c). So, the developed analysis allows the detection as small as 40 pм of cTnI, this value corresponding to $\mathrm{cTnI}$ concentration at small myocardial infarction (13).

Thus, the solid-phase bioluminescent assay we developed made it possible to find DNA aptamers to distinct epitope of cTnI. A couple of the selected aptamers (TnAp2t3 and TnAp10) can be further applied as biospecific molecules not only in bioluminescent, but also in other various assay formats including "label free" ones, which were shown to provide the highest sensitivity (22). At the next step of the study, we plan to conjugate these aptamers through a flexible linker to obtain a single "bivalent" molecule. We assume that the binding affinity of this molecule will essentially exceed that of the initial individual aptamers $(23,24)$.

\section{CONCLUSION}

The current research has demonstrated the use of $\mathrm{Ca}^{2+}$-regulated photoprotein obelin as an instrument to monitor the development of high specific DNA aptamers. Cardiac troponin I was taken as a target.

Obelin-based labels were applied to monitor the course of all conventional stages of aptamer development. They provided fast and easy assessment of DNA library enrichment, relative evaluation of the binding affinity of individual aptamers and their truncated variants, and identification of pairing aptamers. One of the obtained labels, Obe- $\mathrm{dT}_{30}$, is suitable for detection of any molecules containing oligoadenylate and can be applied in the development of other aptamers. Practically simultaneously we have successfully applied the bioluminescent analysis of the kind to evaluate the affinity of novel RNA aptamers to total and glycated hemoglobins (25). The proposed approach allows evaluation of any aptamers in terms of solid-phase assay format, which is preferably used for aptasensor development. Aptamers found with the use of the proposed approach can be further applied as biospecific molecules not only in the bioluminescent but also in other types of analysis. 
Acknowledgements-This work has been supported by the Russian Foundation for Basic Research (RFBR) under the grant no 18-38-00531.

\section{SUPPORTING INFORMATION}

Additional supporting information may be found online in the Supporting Information section at the end of the article:

Figure S1. Secondary structures of aptamer TnAp2 and its truncated variants (RNAfold web server). Probable formation of GQ motif is shown by a dashed line.

Figure S2. SPR sensogram for association and dissociation of TnAp2t1 to recombinant cTnI.

\section{REFERENCES}

1. Kong, H. Y. and J. Byun (2013) Nucleic acid aptamers: new methods for selection, stabilization, and application in biomedical science. Biomol. Ther. (Seoul). 21, 423-434.

2. Jayasena, S. D. (1999) Aptamers: an emerging class of molecules that rival antibodies in diagnostics. Clin. Chem. 45, 1628-1650.

3. Radom, F., P. M. Jurek, M. P. Mazurek, J. Otlewski and F. Jeleń (2013) Aptamers: Molecules of great potential. Biotechnol. Adv. 31, $1260-1274$.

4. Iliuk, A. B., L. Hu and W. A. Tao (2011) Aptamer in bioanalytical applications. Anal. Chem. 83, 4440-4452.

5. Jing, M. and M. T. Bowser (2011) Methods for measuring aptamerprotein equilibria: A review. Anal. Chim. Acta. 686, 9-18.

6. Ryder, S. P., M. I. Recht and J. R. Williamson (2008) Quantitative analysis of protein-RNA interactions by gel mobility shift. Methods Mol. Biol. 488, 99-115.

7. Donaldson, G. P., K. G. Roelofs, Y. Luo, H. O. Sintim and V. T. Lee (2012) A rapid assay for affinity and kinetics of molecular interactions with nucleic acids. Nucleic Acids Res. 40, e48.

8. Ning, Y., W. Li, Y. Duan, M. Yang and L. Deng (2014) High specific DNAzyme-aptamer sensor for Salmonella paratyphi A using single-walled nanotubes-based dual fluorescence-spectrophotometric methods. J. Biomol. Screen. 19, 1099-1106.

9. Apiwat, C., P. Luksirikul, P. Kankla, P. Pongprayoon, K. Treerattrakoon, K. Paiboonsukwong, S. Fucharoen, T. Dharakul and D. Japrung (2016) Graphene based aptasensor for glycated albumin in diabetes mellitus diagnosis and monitoring. Biosens. Bioelectron. 82, 140-145.

10. Liu, Z. J., E. S. Vysotski, C. J. Chen, J. P. Rose, J. Lee and B. C. Wang (2000) Structure of the $\mathrm{Ca}^{2+}$-regulated photoprotein obelin at 1.7 A resolution determined directly from its sulfur substructure. Protein Sci. 9, 2085-2093.

11. Frank, L. A. (2010) $\mathrm{Ca}^{2+}$-regulated photoproteins: Effective immunoassay reporters. Sensors 10, 11287-11300.
12. Frank, L. A. and V. V. Krasitskaya (2014) Application of enzyme bioluminescence for medical diagnostics. Adv. Biochem. Eng. Biotechnol. 144, 175-197.

13. Fathil, M. F., M. K. Md Arshad, S. C. Gopinath, U. Hashim, R. Adzhri, R. M. Ayub, A. R. Ruslinda, M. N. M. Nuzaihan, A. H. Azman, M. Zaki and T. H. Tang (2015) Diagnostics on acute myocardial infarction: Cardiac troponin biomarkers. Biosens. Bioelectron. 70, 209-220.

14. Jo, H., H. Gu, W. Jeon, H. Youn, J. Her, S. K. Kim, J. Lee, J. H. Shin and C. Ban (2015) Electrochemical aptasensor of cardiac troponin I for the early diagnosis of acute myocardial infarction. Anal. Chem. 87, 9869-9875.

15. Dorraj, G. S., M. J. Rassaee, A. M. Latifi, B. Pishgoo and M. Tavallaei (2015) Selection of DNA aptamers against human cardiac troponin I for colorimetric sensor based dot blot application. $J$. Biotechnol. 208, 80-86.

16. Negahdary, M., M. Behjati-Ardakani, N. Sattarahmady, H. Yadegari and H. Heli (2017) Electrochemical aptasensing of human cardiac troponin I based on an array of gold nanodumbbells - Applied to early detection of myocardial infarction. Sens. Actuat. B-Chem. 252, $62-71$.

17. Krasitskaya, V. V., L. P. Burakova, A. A. Komarova, E. E. Bashmakova and L. A. Frank (2017) Mutants of $\mathrm{Ca}^{2+}$-regulated photoprotein obelin for site-specific conjugation. Photochem. Photobiol. 93, 553-557.

18. Frank, L. A., A. I. Petunin and E. S. Vysotski (2004) Bioluminescent immunoassay of thyrotropin and thyroxine using obelin as a label. Anal. Biochem. 305, 240-246.

19. Krasitskaya, V. V., A. N. Kudryavtsev, O. Shimomura and L. A. Frank (2013) Obelin mutants as reporters in bioluminescent dual-analyte binding assay. Anal. Meth. 5, 636-640.

20. Bashmakova, E. E., V. V. Krasitskaya, A. N. Kudryavtsev, V. G Grigorenko and L. A. Frank (2017) Hybrid minimal core streptavidin-obelin as a versatile reporter for bioluminescence-based bioassay. Photochem. Photobiol. 93, 548-552.

21. Burakova, L. P., A. N. Kudryavtsev, G. A. Stepanyuk, I. K. Baykov, V. V. Morozova, N. V. Tikunova, M. A. Dubova, V. N. Lyapustin, V. V. Yakimenko and L. A. Frank (2015) Bioluminescent detection probe for tick-borne encephalitis virus immunoassay. Anal. Bioanal. Chem. 407(18), 5417-5423.

22. Du, Y. and S. Dong (2016) Nucleic acid biosensors: Recent advances and perspectives. Anal Chem. 89, 189-215.

23. Lao, Y. H., K. Peck and L. C. Chen (2009) Enhancement of aptamer microarray sensitivity through spacer optimization and avidity effect. Anal. Chem. 81, 1747-1754.

24. Cho, M., S. S. Oh, J. Nie, R. Stewart, M. J. Radeke, M. Eisenstein, P. J. Coffey, J. A. Thomson and H. T. Soh (2015) Array-based discovery of aptamer pairs. Anal. Chem. 87, 821-828.

25. Davydova, A., M. Vorobyeva, E. Bashmakova, O. Krasheninina, P. Vorobjev, A. Tupikin, M. Kabilov, V. Krasitskaya, L. Frank and A. Venyaminova (2019) Development and characterization of novel $2^{\prime}$ F-RNA aptamers specific to human total and glycated hemoglobins. Anal Biochem. 570, 43-50. 